\title{
A Note Concerning Hamilton Cycles in Some Classes of Grid Graphs
}

\author{
A. N. M. Salman* \\ Faculty of Mathematical Sciences, University of Twente \\ P.O. Box 217, 7500 AE Enschede, The Netherlands \\ salman@math.utwente.nl \\ E. T. Baskoro \\ The Department of Mathematics, ITB \\ Jalan Ganesa 10 Bandung 40132, Indonesia \\ ebaskoro@dns.math.itb.ac.id

\section{H. J. Broersma} \\ Faculty of Mathematical Sciences, University of Twente \\ P.O. Box 217, 7500 AE Enschede, The Netherlands \\ broersma@math.utwente.nl
}

\begin{abstract}
A graph $G$ is called hamiltonian if it contains a Hamilton cycle, i.e. a cycle containing all vertices. Deciding whether a given graph has a Hamilton cycle is an NP-complete problem. But, it is a polynomial problem within some special graph classes. In this paper we consider three classes of grid graphs, namely rectangular grid graph, eta grid graph and omega grid graph. Our main result characterizes which of these graphs are hamiltonian.
\end{abstract}

Keywords: rectangular grid graph, eta grid graph, omega grid graph, Hamilton cycle

AMS Subject Classifications: 05C40, 05C85

${ }^{*}$ Permanent address: The Department of Mathematics, ITB, Jalan Ganesa 10 Bandung 40132, Indonesia 


\section{Introduction}

The infinite grid graph $G^{\infty}$ is defined by the set of vertices $V=\{(x, y) \mid$ $x \in \mathbb{Z}, y \in \mathbb{Z}\}$ and the set of edges $E \subseteq V \times V$ between all pairs of vertices from $V$ at Euclidean distance precisely 1. For any integers $m \geq 1$ and $n \geq 1$, the rectangular grid graph $R(m, n)$ is the (finite) subgraph of $G^{\infty}$ induced by $V(m, n)=\{(x, y) \mid 1 \leq x \leq m, 1 \leq y \leq n, x \in \mathbb{Z}, y \in \mathbb{Z}\}$ (and just containing all edges from $G^{\infty}$ between pairs of vertices from $V(m, n)$ ). This graph $R(m, n)$ is also known as the product graph $P_{m} \times P_{n}$ of two disjoint paths $P_{m}$ and $P_{n}$. For illustration, consider $R(10,7)$ in Figure 1(a). A grid graph is a graph that is isomorphic to a subgraph of $R(m, n)$ induced by a subset of $V(m, n)$ for some integers $m \geq 1$ and $n \geq 1$. It is clear that a grid graph $G=(V, E)$ is a planar graph, i.e. it can be drawn in the plane $\mathbb{R}^{2}$ in such a way that the edges only intersect at the vertices of the graph. In such a drawing, the regions of $\mathbb{R}^{2} \backslash(V \cup E)$ are called the faces of $G$. Exactly one of the faces is unbounded; this is called the outer face; the others are its inner faces. The natural drawing of a grid graph is just described by drawing its vertices in $\mathbb{R}^{2}$ according to their coordinates. A solid grid graph is a grid graph all of whose inner faces have area one (are bounded by a cycle on four vertices) in a natural drawing. A grid graph that is not solid contains inner faces (in a natural drawing) that have area larger than one; these faces are called holes. A Hamilton cycle in a graph $G=(V, E)$ is a cycle containing every vertex of $V$.

Itai, Papadimitriou and Szwarcfiter [?] proved that deciding whether any given grid graph has a Hamilton cycle is an NP-complete problem. But, recently Umans and Lenhart [?] have shown that this problem is polynomial for solid grid graphs, by presenting a complicated algorithm with time complexity $O\left(|V|^{4}\right)$. In this paper we consider three special classes of grid graphs : rectangular grid graphs and two other classes of grid graphs called eta grid graphs and omega grid graphs (which we will define in section 2). Our main result in section 2 characterizes which of the rectangular grid graphs, eta grid graphs and omega grid graphs are hamiltonian and we present explicit constructions of Hamilton cycles.

\section{Eta grid graphs and omega grid graphs}

We now introduce the classes of grid graphs which we call eta grid graphs and omega grid graphs. 


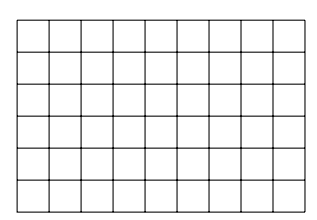

(a)

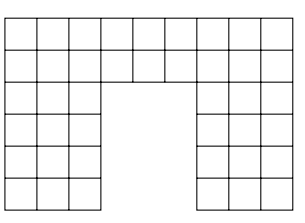

(b)

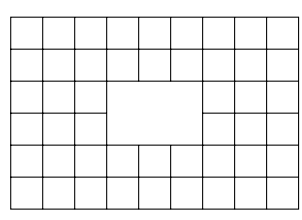

(c)

Figure 1: (a) $R(10,7) \quad$ (b) $\eta(4,3) \quad$ (c) $\varnothing(4,3)$

For $s \geq 3$ and $t \geq 3$ we define an eta grid graph $\eta(s, t)$ a subgraph of $R(3 s-2,3 t-2)$ induced by $V(3 s-2,3 t-2) \backslash\{(x, y) \mid x=s+1, s+$ $2, \cdots, 2 s-2$ and $y=1,2, \cdots, 2 t-2\}$. We define an omega grid graph $\varnothing(s, t)$ as a subgraph of $R(3 s-2,3 t-2)$ induced by $V(3 s-2,3 t-2) \backslash\{(x, y) \mid x=$ $s+1, s+2, \cdots, 2 s-2$ and $y=t+1, t+2, \cdots, 2 t-2\}$. For example, see the eta grid graph $\eta(4,3)$ and the omega grid graph $\varnothing(4,3)$ shown in Figure 1(b) and Figure 1(c), respectively. It is clear from these figures how these graphs should be extended for other values of $m$ and $n$. Notice that in the graphs from these two classes, there is no hole in the eta grid graphs, i.e. they are solid grid graphs, and there is exactly one hole in the omega grid graphs.

The aim of this paper is to prove the following result. We postpone the proofs and constructions (figures) until the section 3 .

Theorem 1 Let $m \geq 2, n \geq 2, s \geq 3$ and $t \geq 3$. Let $R(m, n), \eta(s, t)$ and $\varnothing(s, t)$ denote the rectangular grid graph, the eta grid graph and the omega grid graph as defined above, respectively. Then:

(i) $R(m, n)$ is hamiltonian if and only if $m \cdot n$ is even;

(ii) $\eta(s, t)$ is hamiltonian if and only if $s \cdot t$ is even;

(iii) $\varnothing(s, t)$ is hamiltonian.

\section{Proofs and constructions}

A useful necessary condition for hamiltonicity is the following result due to Grinberg [?].

Lemma 2 Suppose a planar graph $G$ has a Hamilton cycle $H$. Let $G$ be drawn in the plane, and let $r_{i}$ denote the number of faces inside $H$ bounded 
by $i$ edges in this planar embedding. Let $r_{i}^{\prime}$ be the number of faces outside $H$ bounded by $i$ edges. Then the numbers $r_{i}$ and $r_{i}^{\prime}$ satisfy the following equation.

$$
\sum_{i}(i-2)\left(r_{i}-r_{i}^{\prime}\right)=0 .
$$

We use this lemma to show that $R(m, n)$ contains no Hamilton cycle if $m \cdot n$ is odd, and that $\eta(s, t)$ contains no Hamilton cycle if $s \cdot t$ is odd.

Corollary $3 R(m, n)$ contains no Hamilton cycle if $m \cdot n$ is odd.

Proof. There is exactly one face with $2(m+n-2)$ edges and there are exactly $(m-1)(n-1)$ faces with four edges in the planar (natural) drawing of the rectangular grid graph $R(m, n)$. Let this graph be hamiltonian. Then by Lemma 2 we have

$$
(2(m+n-2)-2)(-1)+(4-2)\left(r_{4}-r_{4}^{\prime}\right)=0 .
$$

Hence

$$
r_{4}-r_{4}^{\prime}=m+n-3 .
$$

It is known that the number of faces with four edges is

$$
r_{4}+r_{4}^{\prime}=(m-1)(n-1) .
$$

From equation (1) and (2) we obtain

$$
2 r_{4}=m \cdot n-2 .
$$

So, $m \cdot n$ is even.

Corollary $4 \eta(s, t)$ contains no Hamilton cycle if $s \cdot t$ is odd.

Proof. There is exactly one face with $6(s-1)+10(t-1)$ edges and there are exactly $7(s-1)(t-1)$ faces with four edges in the planar (natural) drawing of the eta grid graph $\eta(s, t)$. Let this graph be hamiltonian. Then by Lemma 2 and using a similar method as in the proof of Corollary 3, we obtain

$$
2 r_{4}=7 s \cdot t-4 s-2 t-2 .
$$

So, $s \cdot t$ is even. 


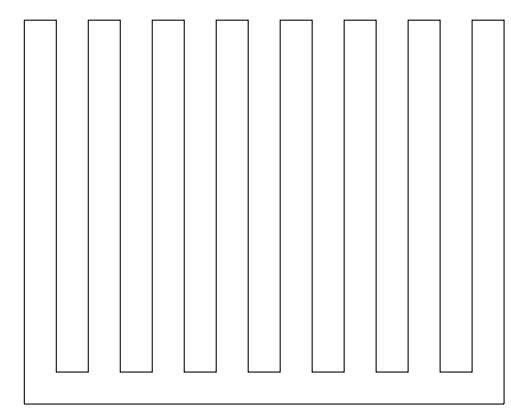

Figure 2: A Hamilton cycle for $R(16,13)$

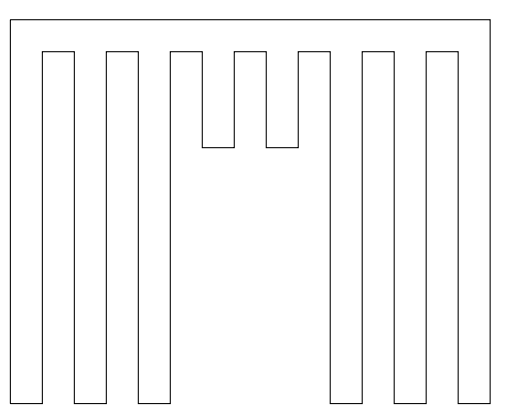

(a)

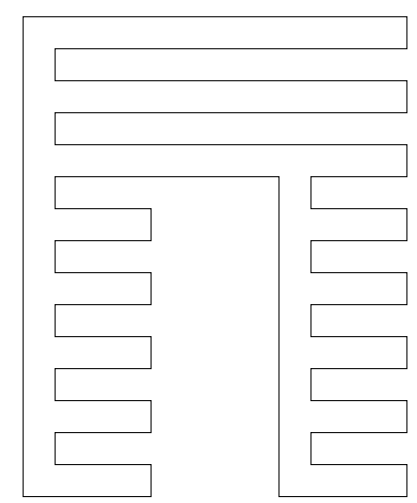

(b)

Figure 3: A Hamilton cycle for : (a) $\eta(6,5) \quad$ (b) $\eta(5,6)$

We complete the proof of Theorem 1 by showing, through construction, the existence of a Hamilton cycle in all cases where $m=16, n=13, s=5$, 6 , or 7 , and $t=5$ or 6 . Meanwhile, for other values of $m, n, s$ and $t$, it is not difficult to see from the patterns in the figures that follow how to extend the solutions.

A Hamilton cycle for $R(16,13)$ is shown in Figure 2. The pattern in this figure can be used for finding a Hamilton cycle for the rectangular grid graph for any even number $m$ or $n$.

Hamilton cycles for $\eta(6,5)$ and $\eta(5,6)$ are shown in Figure 3(a) and 3 (b), respectively. The pattern in Figure 3(a) can be used for finding a Hamilton cycle for the eta grid graph for any even number $m$ and any 


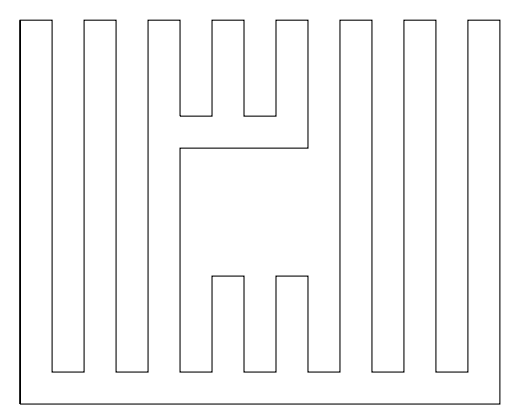

(a)

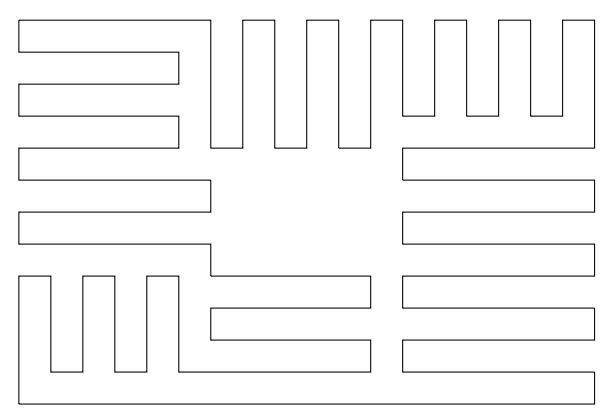

(b)

Figure 4: A Hamilton cycle for : (a) $\varnothing(6,5) \quad$ (b) $\varnothing(7,5)$

number $n$. Meanwhile, the pattern in Figure 3(b) can be used for finding a Hamilton cycle for the eta grid graph for any number $m$ and any even number $n$.

Finally in Figure 4 we show Hamilton cycles for $\varnothing(6,5)$ and $\varnothing(7,5)$. The pattern in Figure 4(a) can be used for finding a Hamilton cycle for the omega grid graph for any even number $m$ or $n$. Meanwhile, the pattern in Figure 4(b) can be used for finding a Hamilton cycle for the omega grid graph for any odd numbers $m$ and $n$.

\section{References}

[1] E.J. Grinberg, Plane homogeneous graphs of degree three without hamiltonian circuits, Latvian Math. Yearbook 5 (1968), 51-58.

[2] A. Itai, C.H. Papadimitriou, and J.L. Szwarcfiter, Hamilton paths in grid graphs, SIAM Journal of Computing 11(4) (1982), 676-686.

[3] C. Umans and W. Lenhart, Hamiltonian cycles in solid grid graphs, Proceedings of the 38th Annual Symposium on Foundations of Computer Science. (1997). 